\section{Tromboembolismo pulmonar y tomografía computarizada helicoidal}

\section{Sr. Director:}

El tromboembolismo pulmonar (TEP) es una patología frecuente de difícil diagnóstico, causante de elevada morbimortalidad. Hasta la fecha varias técnicas de imagen no invasivas (TINI) han sido utilizadas para el diagnóstico de TEP, como la gammagrafía pulmonar de ventilación-perfusión (GVP), la tomografía computarizada (CT) y la resonancia magnética nuclear. A continuación describimos un caso de TEP masivo diagnosticado mediante CT helicoidal (CTH), que evolucionó favorablemente con tratamiento fibrinolítico.

Mujer de 82 años de edad que ingresó en el hospital presentando dolor torácico y 6 horas antes pérdida de conciencia. La exploracíón general y radiografía de tórax fueron normales. El ECG objetivó fibrilación auricular e isquemia subepicárdica anterior. Un ecocardiograma transtorácico demostró aumento de la dimensión del ventrículo derecho, sin trombos. Analíticamente solamente destacaba: gasometría arterial basal: $\mathrm{PH} 7,43, \mathrm{PaCO}_{2} 37,6 \mathrm{mmHg}, \mathrm{PaO}_{2} 64 \mathrm{mmHg}, \mathrm{CO}_{3} \mathrm{H}-25 \mathrm{mEq} / \mathrm{l}$, Sat $\mathrm{O}_{2} 92 \%$. Ante la sospecha de TEP se realizó una CTH que demostró un defecto de repleción subtotal en la arteria pumonar principal derecha (APPD) y oclusión completa del tronco anterior y superior (AS) y de la arteria lobar inferior derecha (ALID), así como oclusión parcial de la arteria lobar inferior izquierda (ALII) y un trombo flotante en la rama segmentaria anterior del lóbulo superior izquierdo (SALSI) (Fig. 1). La gammagrafía de perfusión (GP) objetivó un déficit de perfusión total del pulmón derecho y un déficit de perfusión del segmento lateral y posterior basal izquierdos (PBI). Ya en la UCI, se procedió a administrar $100 \mathrm{mg}$ de rt-pa en 2 horas, mejorando clínica y gasométricamente. La CTH a las 24 horas demostró repermeabilización total de la APPD y casi completa de AS y de la ALII, persistiendo oclusión completa de las ramas segmentarias $7^{\mathrm{a}}$ y $8^{\mathrm{a}}$, así como recanalización completa de ALID y de SALSI. En la GP de control destacaba solamente hipoperfusión en el segmento del lóbulo superior derecho y del PBI. La enferma fue dada de alta a los 5 días.

Desde la descripción de Sinner (1), la evolución tecnológica ha progresado a tal velocidad que la incorporación de CT ultrarrápida como la CTH es actualmente una TINI con alta sensibilidad (98\%)y especificidad (99\%) en relación a la angiografía pulmonar y mayor que la GVP (2-4). Rémy-Jardin y cols. (2) establece que la CTH tiene tres indicaciones básicas: a) diagnóstico de TEP central y de la $2^{\mathrm{a}}$ a la $4^{\mathrm{a}}$ ramas pulmonares, permitiendo una reconstrucción multiplanar; b) TEP crónico complementada con la angiografía pulmonar; c) evolución y seguimiento de pacientes con TEP proximal. Las dificultades diagnósticas residen en la mala visualización de coágulos en las ramas subsegmentarias, a pesar de que en su último estudio visualiza el 50\% de éstas (3), así como las interferencias debidas a nódulos linfáticos intersegmentarios, respiración del paciente y en algunos casos a la persistencia de un agujero oval permeable. La incidencia de émbolos subsegmentarios es del 55,6\% $(3,5)$ y algunos autores han cuestionado la relevancia clínica en pacientes sin enfermedad broncopulmonar y con estudios de trombosis venosa profunda negativos (6). En los algoritmos de diagnóstico de TEP, la primera prueba a realizar es la GVP y la segunda una ecografía venosa de miem- bros inferiores en aquellos casos de GVP con intermedia-baja probabilidad que representa el grupo más frecuente $(4,5,7,8)$. Una prueba positiva de ultrasonidos obligaría a aplicar tratamiento anticoagulante y una negativa, cuya incidencia se ha estimado hasta en un $57 \%$ (9) a realizar una arteriografía pulmonar. Pensamos al igual que otros autores (3,) que la CTH permanece como una TINI indicada para descartar TEP en los casos inconclusos de la GVP, obviando inicialmente la arteriografía y consiguiente morbimortalidad; así como en aquellas situaciones, en que no es posible realizar una GVP y cuando se quieran descartar otros procesos pulmonares o mediastínicos. La rapidez de esta técnica, junto con la alta sensibilidad y especificidad, hace que se pueda utilizar como primera prueba ante la sospecha de TEP y quizás en un futuro no lejano y realizando conjuntamente CT con venografía de miembros inferiores, desplace a la GVP de los algoritmos de diagnóstico $(4,10)$.

M. A. Blasco Navalpotro, M. Soto Ibáñez, A. Málaga López, M. L. Domingo Montañana*

Unidad de Cuidados Intensivos. *Servicio de Radiodiagnóstico. Hospital Universitario Dr. Peset. Valencia

1. Sinner WN. Computed Tomographic patterns of pulmonary thromboembolism and infarction. J Comput Assist Tomogr 1978; 2: 395-399.

2. Remy-Jardin M, Remy J, Wattinne L, Giraud F. Central pulmonary Thromboembolism: Diagnosis with spiral volumetric CT with the single -breath-hold technique. Comparison with pulmonary angiography. Radiology 1992; 185: 381-387.

3. Remy-Jardin M, Rémy J, Deschildre et al. Diagnosi of pulmonary embolism with Spiral CT: Comparison with pulmonary angiography and Scintigraphy. Radiology 1996; 200: 699-706.

4. Gefter W, Hatabu H, Nolland G, Gupta, Henschke C, Palevsky H. Pulmonary Thromboembolism: Recent developments in diagnosis with CT and MR imaging. Radiology 1995; 197: 561-574.

5. PIOPED investigator. Value of the ventilation/perfusion scan in acute pulmonary embolism : results of the prospective investigation of pulmonary embolism diagnosis PIOPED. JAMA 1990; 263: 2753-2759.

6. Hull RD, Raskob GE, Coates G, Panjv AA, Gill GJ. A new noninvasive management strategy for patients with supected pulmonary ebolism. 
Arch Intern Med 1989; 149: 2549-2555.

7. Ginsberg JS. Management of venous thromboembolism. N Engl J Med 1996: 335(24):1816-1828.

8. Hull RD, Raskob GE, Ginsberg JS et al. A noninvaive strategy for the treatment of patient with suspected pulmonary embolism. Arch Intern Med 1994; 154: 289-297.

9. Hull RD, Nirh J, Carter CJ, et al. Pulmonary angiography ventilation lung Scanning and venography for clinically suspected pulmonary embolism with abnormal perfuion lung Scan. Ann Intern Med 1983; 98 891-9.

10. Stehling MK, Rosen MP, Weintraub J, Km D, Raptopoulos V. Spiral CT venography of the lower extremity. AJR 1994; 163: 451-453.

\section{Síndrome de Sjögren primario asociado a hepatitis autoinmune}

\section{Sr. Director:}

El síndrome de Sjögren (SS) es una enfermedad autoinmune de etiología desconocida, caracterizada por la infiltración linfocitaria de las glándulas lagrimales y salivares, provocando queratoconjuntivitis seca y xerostomía. Sin embargo, puede existir además afectación de otros órganos y aparatos. En el tubo digestivo se puede afectar no sólo la boca, sino también las glándulas exocrinas del esófago, estómago, intestino delgado y grueso (1). En el tracto hepatobiliar el SS puede asociarse con frecuencia a cirrosis biliar primaria y más raramente a colangitis esclerosante (1). Presentamos un caso de síndrome de Sjögren asociado a hepatitis crónica autoinmune, fenómeno conocido aunque poco frecuente.

Mujer de 34 años sin antecedentes de interés que consultó por sequedad de boca y sensación de cuerpo extraño en ambos ojos de 3 meses de evolución. La paciente no refería fotosensibilidad, eritema, fenómeno de Raynaud, úlceras orales o genitales, artritis, artralgias, fiebre, sudoración o pérdida de peso. La exploración física era normal. En el hemograma existía una leucocitosis mantenida con linfocitosis 13.590 leucocitos $/ \mathrm{mm}^{3}, 17 \%$ neutrófilos, $76 \%$ linfocitos, 4 monocitos, $2 \%$ eosinófilos, $1 \%$ basófilos, siendo la serie roja y las plaquetas normales. La velocidad de sedientación globular fue de 12 $\mathrm{mm}$ a la $1^{\mathrm{a}}$ hora. Las concentraciones plasmáticas de glucosa, urea, creatinina, sodio, potasio, colesterol total, triglicéridos, bilirrubina, TSH y $\mathrm{T}_{4}$ libre se encontraban dentro de los límites normales. Asimismo, el proteinograma y el estudio de coagulación fueron normales. La AST fue de 132 UI/l (normal: < $35 \mathrm{U} / \mathrm{l}$ ), la ALT de $138 \mathrm{U} / 1$ (normal: < $35 \mathrm{U} / \mathrm{L}$ ), la gamaglutamiltransferasa de $100 \mathrm{U} / 1$ (normal: < $52 \mathrm{U} / 1$ ) y la fosfatasa alcalina de $859 \mathrm{UI} / 1$ (normal < $250 \mathrm{U} / \mathrm{l}$ ). Las concentraciones

\section{TABLA I}

EVO LUCIÓN DE LAS ENZIM AS HEPÁTICAS EN PLASM A EN NUESTRA PACIENTE (VALO RES BASALES Y TRAS TRATAM IENTO CON DEFLAZACORT 30 M G/DÍA)

\begin{tabular}{lccccc}
\hline & BASAL & L $^{\text {ER }}$ M ES & $2^{\circ}$ M ES & $3^{\text {ER }}$ M ES & $5^{\circ}$ M ES \\
\hline AST (U/L) & 132 & 65 & 54 & 52 & 38 \\
ALT (U/L) & 138 & 85 & 72 & 70 & 42 \\
FA (U/L) & 859 & 511 & 367 & 307 & 208 \\
GGT (U/L) & 100 & 82 & 74 & 56 & 28 \\
\hline
\end{tabular}

ALT: Alanino aminotranserasa. AST: Aspartato aminotransferasa. FA: Fosfatasa alcalina. GGT: gamma-glutamil-transpeptidasa. plasmáticas de hierro, ferritina, ceruloplasmina y alfa-1-antitripsina fueron normales. Asimismo, la serología del virus de la hepatitis $\mathrm{B}$ y $\mathrm{C}$, así como de lúes, rubéola, brucella, citomegalovirus y mononucleosis fueron negativas. Los anticuerpos antinucleares (ANA fueron positivos a un título de 1160, con un patrón mixto. El factor reumatoide y los autoanticuerpos anti DNA, Anti RNP, Anti Ro, Anti LA, Scl-70, anti Jo-1, antimitocondriales (AMA), anti músculo liso (SMA), anticélulas $\mathrm{P}$ y anti LKM-l fueron negativos. La radiografía posteroanterior de tórax y la ecografía abdominal no mostraron alteraciones patológicas. El test de Schirmer bilateral fue de $5 \mathrm{~mm}$ a los 5 minutos (normal > $10 \mathrm{~mm}$ ). En la biopsia de labio se observa a nivel de glándulas salivares atrofia acinar y denso infiltrado crónico linfocitario (grado III según la escala de Chisholm-Mason) (3). La punción-biopsia hepática con aguja de Tru-cut bajo control ecográfico, mostró unos canalículos biliares normales, necrosis hepatocitaria periportal, marcado infiltrado inflamatorio linfocitario con fibrosis subyacente en torno a dicho espacio y a nivel del lobulillo hepático, y tendencia a la formación de rosetas (Índice de Knodell: 12/22), todo ello compatible con hepatitis crónica activa. Iniciamos tratamiento con deflazacort a dosis de $30 \mathrm{mg}$ /día durante 2 meses y reducción progresiva en los parámetros bioquímicos hepáticos con el uso terapéutico del deflazacort en nuestra paciente. Habitualmente la respuesta a los corticosteroides ocurre a los pocos meses del inicio del tratamiento, debiéndose reducir la dosis progresivamente $(8,9)$. No existe, sin embargo, una guía establecida de cómo reducir dicha dosis. Además, la retirada del fármaco suele acompañarse de una recidiva del proceso hepático. En tales casos, la azatioprina a dosis de 50-150 mg/día sola o bien asociada a bajas dosis de corticosteroides, parece la opción más recomendable y efectiva $(8,10)$

\section{J. L. Zambrana García, F. Gallego Rojo, G. Cruz Caparrós, F. Díez García}

Servicio de Medicina Interna. Hospital de Poniente. El Ejido. Almería

1. Sheikh SH, Shaw-Stiffel TA. The gastrointestinal manifestations of Sjogren's syndrome. Am J Gastroenterol 1995; 90:9-14.

2. Wada T, Motoo Y, Ohmizo R, et al. Association of mixed connective tissue disease. Sjogren's syndrome and autoimmune hepatitis: Report of a case. Jpn J Med 1991; 30:278-280.

3. Fox RI, Robinson CA, Curd JG, Kosin F, Howell FV. Sjogren's syndrome. Proposed criteria for classification. Arthritis Rheum 1986; 29:577584.

4. Bloch KJ, Buchanan WW, Wohl MJ, Bunim JJ. Sjogren's syndrome: A clinical, pathological and serological study of 62 cases. Medicine (Baltimore) $1965 ; 44: 187-231$

5. Crowe JP, Christensen E, Butler J, et al. Primary biliary cirrhosis: The prealence of hypothyroidism and its relationship to thyroid autoantibodies and sicca syndrome. Gastroenterology 1980; 78:14371441.

6. Johnson PJ, McFarlane IG. Meeting report: International Autoimmune Hepatitis Group. Hepatology 1993; 18:998-1005.

7. Aiza I, Schiff ER. Autoimmune hepatitis. Curr Opin Gastroenterol $1995 ; 11: 238-244$

8. Krawitt EL. Autoimmune hepatitis. N Engl J Med 1996; 14:897903.

9. Sánchez-Urdazpal LS, Czaja AJ, van Hoek B, grom RAF, Wiesner RH. Prognostic features and role of livér transplantation in severe corticosteroid-treated autoimmune chronic active hepatitis. Hepatology 1992. 15:215-221.

10. Johnson PJ, McFarlane IG, Williams R. Azathioprine for long-term maintenance of remission in autoimmune hepatitis. N Engl J Med 1995; 333:958-963. 